\title{
CLASSIFICATION OF SINGULARITIES FOR BLOWING UP SOLUTIONS IN HIGHER DIMENSIONS
}

\author{
J. J. L. VELÁZQUEZ
}

ABstract. Consider the Cauchy problem

(P) $\begin{cases}u_{t}-\Delta u=u^{p} & \text { when } x \in \mathbb{R}^{N}, t>0, N \geq 1, \\ u(x, 0)=u_{0}(x) & \text { when } x \in \mathbb{R}^{N},\end{cases}$

where $p>1$, and $u_{0}(x)$ is a continuous, nonnegative and bounded function. It is known that, under fairly general assumptions on $u_{0}(x)$, the unique solution of $(\mathrm{P}), u(x, t)$, blows up in a finite time, by which we mean that

$$
\limsup _{t \uparrow T}\left(\sup _{x \in \mathbb{R}^{N}} u(x, t)\right)=+\infty .
$$

In this paper we shall assume that $u(x, t)$ blows up at $x=0, t=T<+\infty$, and derive the possible asymptotic behaviours of $u(x, t)$ as $(x, t) \rightarrow(0, T)$, under general assumptions on the blow-up rate.

\section{INTRODUCTION AND DESCRIPTION OF RESULTS}

This paper deals with the following problem:

$$
\begin{gathered}
u_{t}-\Delta u=u^{p} \quad \text { when } x \in \mathbb{R}^{N}, t>0, p>1, \\
u(x, 0)=u_{0}(x) \quad \text { when } x \in \mathbb{R}^{N}
\end{gathered}
$$

where $u_{0}(x)$ is a continuous, nonnegative and bounded function. Local (in time) existence of a classical solution $u(x, t)$ of (1.1), (1.2) follows at once from standard results. It is said that $u(x, t)$ blows up in a finite time $T<+\infty$, if $u(x, t)$ satisfies $(1.1),(1.2)$ in $\mathbb{R}^{N} \times(0, T)$ and

$$
\limsup _{t \uparrow T}\left(\sup _{x \in \mathbb{R}^{N}} u(x, t)\right)=+\infty .
$$

In such case, a point $x_{0} \in \mathbb{R}^{N}$ is called a blow-up point of $u(x, t)$ if there exist sequences $\left\{x_{n}\right\},\left\{t_{n}\right\}$ such that $\lim _{n \rightarrow \infty} x_{n}=x_{0}, \lim _{n \rightarrow \infty} t_{n}=T$, and $\lim _{n \rightarrow \infty} u\left(x_{n}, t_{n}\right)=\infty$. Conditions on $u_{0}(x)$ and $p$ under which $u(x, t)$ blows up in finite time have been extensively discussed in the literature (cf., for instance, [Fu, AW]). See also [BBE, CM, FM, L, W] for related results.

Received by the editors April 29, 1991.

1991 Mathematics Subject Classification. Primary 35B40, 35K55, 35K57.

Key words and phrases. Semilinear diffusion equations, asymptotic behaviour, classification of singularities, blow-up.

Partially supported by CICYT Research Grant PB90-0235 and EEC Contract SC1-0019C. 
We shall concern ourselves with the task of describing the asymptotics of solutions near blow-up points. To this end, we shall assume henceforth that

$$
u(x, t) \text { blows up at } x=0 \text { and } t=T<+\infty .
$$

Moreover, some information about the manner in which blow-up happens will be taken for granted. Namely, we will suppose throughout that

$$
\begin{gathered}
u(x, t) \leq M(T-t)^{-1 /(p-1)} \begin{array}{l}
\text { for any } x \in \mathbb{R}^{N}, t<T, \text { and } \\
\text { some constant } M .
\end{array} \\
\lim _{t \rightarrow T}(T-t)^{1 /(p-1)} u\left(x(T-t)^{1 / 2}, t\right)=(p-1)^{-1 /(p-1)}, \\
\text { uniformly on bounded sets }|x| \leq R \text { with } R>0 .
\end{gathered}
$$

We remark in passing that (1.4) holds under loose assumptions on the initial values $u_{0}(x)$ when $p<(N+2) /(N-2)$; see for instance [GP] for the case $N=1$ and the series of fundamental papers [GK1, GK2, GK3], where no restriction on the dimension $N$ is made.

Our aim here consists in obtaining an additional term in the asymptotic expansion in (1.4b) when $N>1$. Besides its intrinsic interest, we expect that this fact will be important in describing the local structure of the blow-up set, as it happens to be in the one-dimensional case (cf. [HV1, HV2, HV3]). We shall recall briefly the corresponding results in [HV1, HV2], since these are relevant for the analysis to be performed presently. Following [GP and GK1], we introduce similarity variables

(1.5) $u(x, t)=(T-t)^{-1 /(p-1)} \phi(y, \tau) \quad$ where $y=\frac{x}{\sqrt{T-t}}, \tau=-\log (T-t)$, so that $\phi$ satisfies

$$
\phi_{\tau}=\phi_{y y}-y \phi_{y} / 2+\phi+f_{1}(\phi)
$$

where

$$
f_{1}(\phi)=\phi^{p}-\frac{p}{p-1} \phi
$$

We then linearize about the nontrivial stationary solution of (1.6) by setting

$$
\phi(y, \tau)=(p-1)^{-1 /(p-1)}+\Psi(y, \tau)
$$

so that $\Psi(y, \tau)$ solves

$$
\Psi_{\tau}=\Psi_{y y}-y \Psi_{y} / 2+\Psi+f(\Psi) \equiv A \Psi+f(\Psi),
$$

where

$$
f(\Psi)=\left((p-1)^{-1 /(p-1)}+\Psi\right)^{p}-(p-1)^{-p /(p-1)}-\frac{p \Psi}{p-1}
$$

and $f(s)=O\left(s^{2}\right)$ as $s \rightarrow 0$. Here and henceforth, free use will be made of the customary asymptotic notations $o(), O(), \ll, \approx$, etc. For $1 \leq q,+\infty$ and any positive integer $k \geq 1$, we define the spaces

$$
L_{w}^{q}(\mathbb{R})=\left\{g \in L_{\mathrm{loc}}^{q}: \int_{\mathbb{R}}|g(s)|^{q} e^{-s^{2} / 4} d s<+\infty\right\},
$$




$$
\begin{array}{r}
H_{w}^{k}(\mathbb{R})=\left\{g \in L_{\text {loc }}^{2}(\mathbb{R}): \text { for any } j \in[0, k], g^{(j)} \in L_{\text {loc }}^{2}(\mathbb{R})\right. \\
\text { and } \left.\int_{\mathbb{R}}\left|g^{(j)}(s)\right|^{2} e^{-s^{2} / 4} d s<+\infty\right\} .
\end{array}
$$

It is readily seen that $L_{w}^{2}(\mathbb{R})$ (resp. $L_{w}^{q}(\mathbb{R}), 1 \leq q<+\infty, q \neq 2$ ) is a Hilbert space (resp. a Banach space) when endowed with the norm

$$
\|g\|_{2, w}^{2} \equiv\langle g, g\rangle=\int_{\mathbb{R}}|g(s)|^{2} e^{-s^{2} / 4} d s
$$

(resp.

$$
\left.\|g\|_{q, w}^{q}=\int_{\mathbb{R}}|g(s)|^{q} e^{-s^{2} / 4} d s\right) .
$$

Since the $L_{w}^{2}$ norm will be extensively used hereafter, we shall denote it by \|\| for simplicity. Clearly, for $k \geq 1, H_{w}^{k}(\mathbb{R})$ can be given a structure of Hilbert space in a straightforward way. It is then natural to consider (1.8) as a dynamical system in $L_{w}^{2}(\mathbb{R})$. Actually, operator $A$ defined in (1.8) with domain $D(A)=H_{w}^{2}(\mathbb{R})$ is selfadjoint in $L_{w}^{2}(\mathbb{R})$ and has eigenvalues $\lambda_{n}=1-n / 2$, $n=0,1,2, \ldots$, with eigenfunctions $H_{n}(y)$ given by

$$
H_{n}(y)=c_{n} \widetilde{H}_{n}(y / 2), \text { where } c_{n}=\left(2^{n / 2}(4 \pi)^{1 / 4}(n !)^{1 / 2}\right)^{-1},
$$

and $\widetilde{H}_{n}(y)$ is the standard $n$th Hermite polynomial,

$$
\text { so that }\left\|H_{n}\right\|=1 \text { for any } n \text {. }
$$

The following result was proved in [HV1] and [HV2].

Theorem A. Assume that $N=1$ and (1.3) holds. Then one of the following cases occurs:

$$
\begin{gathered}
\Psi(\cdot, \tau) \equiv 0 \text { for any } \tau>0 . \\
\Psi(\cdot, \tau)+\frac{(4 \pi)^{1 / 4}(p-1)^{-1 /(p-1)}}{\sqrt{2} p} \cdot \frac{H_{2}(y)}{\tau}=o\left(\frac{1}{\tau}\right) \quad \text { as } \tau \rightarrow \infty .
\end{gathered}
$$

(1.11c) There exist $m$ even, $m \geq 4$, and $C \neq 0$ such that

$$
\Psi(\cdot, \tau)-C e^{(1-m / 2) \tau} H_{m}(\cdot)=o\left(e^{(1-m / 2) \tau}\right) \text { as } \tau \rightarrow \infty,
$$

where convergence takes place in $H_{u}^{1}$ as well as in $C_{\mathrm{loc}}^{k, \alpha}$ for any $k \geq 0$ and $\alpha \in(0,1)$.

To deal with the case $N>1$, we modify our functional frame in a natural way. Let $q, k$ be as in (1.9), and set

$$
L_{w}^{q}\left(\mathbb{R}^{N}\right)=\left\{f \in L_{\mathrm{loc}}^{q}\left(\mathbb{R}^{N}\right): \int_{\mathbb{R}}|f(s)|^{q} e^{-s^{2} / 4} d s<+\infty\right\},
$$

$$
\begin{array}{r}
H_{w}^{k}\left(\mathbb{R}^{N}\right)=\left\{g \in L_{w}^{2}\left(\mathbb{R}^{N}\right): \frac{\partial^{\alpha} f}{\partial x^{\alpha}} \in L_{w}^{2}\left(\mathbb{R}^{N}\right) \text { where } \alpha=\left(\alpha_{1}, \ldots, \alpha_{N}\right)\right. \\
\left.|\alpha|=\alpha_{1}+\cdots+\alpha_{N} \leq k, \text { and } \frac{\partial^{\alpha} f}{\partial x^{\alpha}}=\frac{\partial^{|\alpha|} f}{\partial x_{1}^{\left(\alpha_{1}\right.} \cdots \partial x_{N}^{\left(\alpha_{N}\right.}}\right\} .
\end{array}
$$


The change of variables; (1.5), (1.7) leads then to

$$
\begin{gathered}
\Psi_{\tau}=A_{N} \Psi+f(\Psi), \quad \text { where } f(\Psi)=O\left(\Psi^{2}\right) \text { as } \Psi \rightarrow 0 \text { and } \\
A_{N}=\Delta \Psi-\frac{y \cdot \nabla \Psi}{2}+\Psi .
\end{gathered}
$$

Operator $A_{N}$ is now selfadjoint in $L_{w}^{2}\left(\mathbb{R}^{N}\right)$ with domain $D\left(A_{N}\right)=H_{w}^{2}\left(\mathbb{R}^{N}\right)$. Its spectrum consists of the eigenvalues

$$
1-\frac{m_{1}+\cdots+m_{N}}{2} \text { where } m_{1}, m_{2}, \ldots, m_{N}=0,1,2, \ldots,
$$

and the corresponding eigenfunctions are

$$
\begin{aligned}
H_{m_{1}}, m_{2}, \ldots, m_{N} & =H_{m_{1}}\left(y_{1}\right) \cdots H_{m_{N}}\left(y_{N}\right), \\
& \text { where } H_{j}\left(y_{j}\right) \text { is defined in (1.10). }
\end{aligned}
$$

For $x=\left(x_{1}, \ldots, x_{N}\right)$ and $\alpha=\left(\alpha_{1}, \ldots, \alpha_{N}\right)$, let us write $x^{\alpha}=x_{1}^{\alpha_{1}} \cdots x_{N}^{\alpha_{N}}$, and $H_{\alpha}(x)=H_{\alpha_{1}}\left(x_{1}\right) \cdots H_{\alpha_{N}}\left(x_{N}\right)$. Our main result is

Theorem. Let $u(x, t)$ be the solution of (1.1), (1.2) and assume that (1.3), (1.4) hold. Let $\Psi(\cdot, \tau)$ be given by (1.5), (1.7). Then, if $\Psi(\cdot, \tau) \not \equiv 0$ for some $\tau>0$, the following possibilities arise. Either there exists an orthogonal transformation of coordinate axes such that, denoting still by $y$ the new coordinates

$$
\Psi(\cdot, \tau)=-\frac{C_{p}}{\tau} \sum_{k=1}^{l} H_{2}\left(y_{k}\right)+o\left(\frac{1}{\tau}\right) \quad \text { as } \tau \rightarrow \infty
$$

where $1 \leq l \leq N$ and

$$
C_{p}=(4 \pi)^{1 / 4}(p-1)^{-1 /(p-1)} / \sqrt{2} p
$$

or there exists an even number $m, m \geq 4$, and constants $c_{\alpha}$ not all zero such that

$$
\Psi(\cdot, \tau)=-e^{(1-m / 2) \tau} \sum_{|\alpha|=m} c_{\alpha} H_{\alpha}(y)+o\left(e^{(1-m / 2) \tau}\right) \quad \text { as } \tau \rightarrow \infty,
$$

where the homogeneous multilinear form

$$
B(x)=\sum_{|\alpha|=m} c_{\alpha} x^{\alpha}
$$

is nonnegative. In cases $(1.15 \mathrm{a}),(1.15 \mathrm{~b})$ convergence takes place in $H_{w}^{1}\left(\mathbb{R}^{N}\right)$ as well as in $C_{\mathrm{loc}}^{k, \alpha}\left(\mathbb{R}^{N}\right)$ for any $k \geq 0$ and $\alpha \in(0,1)$.

We next discuss briefly previous work to ours, as well as some related results. In [B], the author considers the Cauchy-Dirichlet problem for

$$
u_{t}-\Delta u=e^{u}
$$

in a bounded domain with homogeneous side conditions. Such a choice of the reaction term $f(u)=e^{u}$ is well known in combustion theory (cf. for instance $[\mathrm{BE}]$ ). It is shown in [B] that solutions satisfying (1.15a) (or rather its counterpart for (1.16)) actually exist. 
On the other hand, let us denote by $\left\{e^{+}\right\}_{j=1}^{k}$ the eigenfunctions of $A_{N}$ corresponding to positive eigenvalues (cf. (1.14)), $\left\{e_{j}^{0}\right\}_{j=1}^{m}$ its eigenvalues with eigenvalue zero, and $\left\{e_{j}^{-}\right\}_{j=1}^{\infty}$ its eigenfunctions with negative eigenvalues. Recalling the definition of $\Psi(y, \tau)$ in (1.7), we may write

$$
\Psi(y, \tau)=\sum_{j=1}^{k} \beta_{j}(\tau) e_{j}^{+}(y)+\sum_{j=1}^{m} \alpha_{j}(\tau) e_{j}^{0}(y)+\sum_{j=1}^{\infty} \gamma_{j}(\tau) e_{j}^{-}(y) .
$$

Then the following result has been shown in [FK].

(1.17) Either $\Psi \rightarrow 0$ exponentially fast as $\tau \rightarrow \infty$, or for any $\varepsilon>0$ there is a time $s_{0}$ such that

$$
\sum_{j=1}^{k} \beta_{j}^{2}(\tau)+\sum_{j=1}^{\infty} \gamma_{j}^{2}(\tau) \leq \varepsilon \sum_{j=1}^{m} \alpha_{j}^{2}(\tau) \quad \text { for } \tau \geq s_{0} .
$$

Moreover, if $\Psi$ does not approach zero exponentially fast, the neutral modes $\left\{\alpha_{j}\right\}_{j=1}^{m}$ satisfy

$$
\alpha_{j}=\frac{p}{2}(p-1)^{1 /(p-1)} \Pi_{j}^{0}\left(\Psi_{0}^{2}\right)+O\left(\varepsilon \sum_{j=1}^{m} \alpha_{j}^{2}\right)
$$

where $\Pi_{j}^{0}$ denotes orthogonal projection onto $e_{j}^{0}$ and $\Psi_{0}$ is the neutral component of $\Psi$ :

$$
\Psi_{0}=\sum_{j=1}^{m} \alpha_{j}(\tau) e_{j}^{0}(y)
$$

Concerning the alternative state in (1.17), we obtain here a precise description of the situation where $\Psi(\cdot, \tau) \rightarrow 0$ as $\tau \rightarrow \infty$ exponentially fast (cf. $(1.15 \mathrm{~b}))$. Furthermore, an asymptotic expansion for $\Psi(\cdot, \tau)$ in the case where the neutral modes prevail is given in (1.15a). It is worthwhile to point out that our approach is technically rather different from that in [FK]. While those authors rely heavily on a center manifold viewpoint, we proceed along the lines of the perturbative techniques already used in [HV1, HV2, HV3] to deal with the one-dimensional case. The techniques introduced in these works, as well as those developed in [FK], have been applied in [HV5, BB, Li]. Of these, [HV5] and [BB] deal with the combustion model (1.16). In [HV5], the final blow-up profiles for the corresponding Cauchy problem are obtained, and the existence of flat blow-up structures (in the sense of $(1.11 \mathrm{c})$ ) is shown in a particular case, which correspond to two maxima collapsing at blow-up time. The paper [BB] is concerned with the description of final blow-up profiles for radial solutions in any space dimension, under some assumptions on the initial values which are not required in [HV5].

After completion of this article, we learned about related and independent work by Filippas and Liu [FL]. In that paper, the authors obtain, among other results, that either $(1.15 \mathrm{a})$ holds, or the scaled error $\Psi(y, \tau)$ must decay at least exponentially as $\tau \rightarrow \infty$. However, decays faster than exponential are not excluded, and no precise formula like $(1.15 \mathrm{~b})$ is obtained therein. 
The plan of this paper is as follows. A number of auxiliary results which extend previously known facts for the case $N=1$ can be found in $\S 2$. The main novel points in the proof of our main result are then discussed in $\S 3$.

\section{Preliminaries}

In this section we shall gather some results which are analogous to those previously obtained in [HV1] for the case $N=1$. To keep this paper within reasonable bounds, we shall just stress the points where relevant differences appear with respect to the corresponding results in [HV1], and refer to that work for details.

As a starting point, we state a crucial delayed regularizing effect (cf. [HV1, $\S 2])$.

Lemma 2.1. Assume that $\Psi(y, \tau)$ satisfies (1.13) and $|\Psi| \leq M<\infty$ for some $M>0$. Then for any $r>1, q>1$, and $L>0$ there exist $\tau_{0}^{*}=\tau_{0}^{*}(q, r)$ and $C=C(N, r, q, L)$ such that

(2.1) $\left\|\Psi\left(\cdot, \tau+\tau^{*}\right)\right\|_{r, w} \leq C\|\Psi(\cdot, \tau)\|_{q, w} \quad$ for any $\tau \geq 0$ and $\tau^{*} \in\left[\tau_{0}^{*}, \tau_{0}^{*}+L\right]$.

It is worthwhile to point out here that $(2.1)$ is basically a linear effect, which holds indeed for solutions of the heat equation. To proceed further, we notice that, since the set $\left\{H_{\alpha}: \alpha \in \mathbb{N}^{N}\right\}$ is an orthonormal basis in $L_{w}^{2}\left(\mathbb{R}^{N}\right)$, we can represent $\Psi$ as

$$
\Psi(y, \tau)=\sum_{\alpha} a_{\alpha}(\tau) H_{\alpha}(y)
$$

for some coefficients $a_{\alpha}(\tau)$. The following nondegeneracy result can be proved exactly as in [HV1, §3].

Lemma 2.2. Assume that $|\Psi(y, \tau)|$ is bounded. Suppose also that for any $R>0$ there exists $C=C(R)$ such that

$$
\|\Psi(\cdot, \tau)\| \leq C e^{-R \tau} \quad \text { for } \tau \geq 0 .
$$

Then $\Psi(y, \tau) \equiv 0$.

As a further step, we notice that the first modes in (2.2) represent negligible contributions to the $L_{w}^{2}$-norm of $\Psi$.

Lemma 2.3. Let $\Psi(y, \tau)$ be as in the previous lemmata, and assume also that $\lim _{\tau \rightarrow \infty}\|\Psi(\cdot, \tau)\|=0$. Then there holds

$$
\lim _{\tau \rightarrow \infty} \frac{\sum_{|\alpha| \leq 1}\left|a_{\alpha}(\tau)\right|}{\|\Psi(\cdot, \tau)\|}=0 .
$$

Proof. It consists in a suitable modification of that of Proposition 4.1 in [HV1]. Of the various estimates which are used there to derive (2.3) when $N=1$, only one does not carry over as such when $N>1$, namely that obtained in Lemma 4.4. To circumvent this problem we proceed as follows. Assume that (2.3) does not hold, so that there exists a sequence $\left\{\tau_{j}\right\}$ with $\lim _{j \rightarrow \infty} \tau_{j}=\infty$ and a constant $\varepsilon>0$ such that

$$
\sum_{|\alpha| \leq 1}\left|a_{\alpha}\left(\tau_{j}\right)\right| \geq \varepsilon\left\|\Psi\left(\cdot, \tau_{j}\right)\right\| .
$$


Set now $\Psi(y, \tau)=\sigma_{j}(y, \tau)+\omega_{j}(y, \tau), j=1,2, \ldots$, where for any such $j$, $\sigma_{j}$ solves

$$
\begin{gathered}
\left(\sigma_{j}\right)_{\tau}=\Delta\left(\sigma_{j}\right)-\frac{y}{2} \cdot \nabla\left(\sigma_{j}\right)+\sigma_{j} \quad \text { if } \tau>\tau_{j}, \\
\sigma_{j}\left(y, \tau_{j}\right)=\Psi\left(y, \tau_{j}\right) .
\end{gathered}
$$

For $R>0$, let us define $\chi_{R}(y)$ by $\chi_{R}(y)=1$ if $|y|<R$ and zero otherwise. We then have

(2.4) For any given $L>0, \varepsilon>0$, and $R>0$ there exists $C>0$ independent of $R, \varepsilon$, and a constant $C_{\varepsilon}$ such that

$$
\begin{gathered}
\frac{1}{\left\|\Psi\left(\cdot, \tau_{j}\right)\right\|}\left(\int_{\tau_{j}}^{\tau_{j}+L}\left\|\chi_{R}(\cdot) g\left(\left|\sigma_{j}(\cdot, s)\right|\right)\right\|^{2} d s\right)^{1 / 2} \\
\leq C \varepsilon+C_{\varepsilon}\left\|\Psi\left(\cdot, \tau_{j}\right)\right\|^{1 / 4}\left(1+e^{C R^{2}}\right)^{5 / 4}
\end{gathered}
$$

where $g(s)=\min \left(s, s^{5 / 4}\right)$

To obtain (2.4), we notice that

$$
\begin{aligned}
\int_{\tau_{j}}^{\tau_{j}+L} & \left\|\chi_{R}(\cdot) g\left(\left|\sigma_{j}(\cdot, s)\right|\right)\right\|^{2} d s=\int_{\tau_{j}}^{\tau_{j}+\varepsilon}()+\int_{\tau_{j}+\varepsilon}^{\tau_{j}+L}() \\
\leq & C \int_{\tau_{j}}^{\tau_{j}+\varepsilon}\left\|\chi_{R}(\cdot)\left|\sigma_{j}(\cdot, s)\right|\right\|^{2} d s+\int_{\tau_{j}+\varepsilon}^{\tau_{j}+L}\left\|\chi_{R}(\cdot)\left|\sigma_{j}(\cdot, s)\right|^{5 / 4}\right\|^{2} d s \\
& \equiv I_{1}+I_{2} .
\end{aligned}
$$

We then estimate $I_{1}$ as follows:

$$
\begin{aligned}
I_{1} \leq & C e^{2\left(\tau-\tau_{j}\right)} \int_{\tau_{j}}^{\tau_{j}+\varepsilon} d s \int_{\mathbb{R}^{N}} e^{-\xi^{2} / 4} \\
& \times\left(\int_{\mathbb{R}^{N}} \frac{\exp \left(-\left(\xi e^{-\left(s-\tau_{j}\right) / 2}-\lambda\right)^{2} / 4\left(1-e^{-\left(\tau-\tau_{j}\right)}\right)\right.}{\left(4 \pi\left(1-e^{-\left(s-\tau_{j}\right)}\right)\right)^{N / 2}}\left|\Psi\left(\lambda, \tau_{j}\right)\right| d \lambda\right)^{2} d \xi \\
\leq & C e^{2 L} \int_{\tau_{j}}^{\tau_{j}+\varepsilon} d s \int_{\mathbb{R}^{N}}\left|\Psi\left(\lambda, \tau_{j}\right)\right|^{2} e^{-\lambda^{2} / 4} \\
& \times\left(\int_{\mathbb{R}^{N}} \frac{\exp \left(-\left(\xi e^{-\left(s-\tau_{j}\right) / 2}-\lambda\right)^{2} / 4\left(1-e^{-\left(\tau-\tau_{j}\right)}\right)-\xi^{2} / 4+\lambda^{2} / 4\right)}{\left(4 \pi\left(1-e^{-\left(s-\tau_{j}\right)}\right)\right)^{N / 2}} d \lambda\right) d \xi \\
\leq & C \varepsilon\left\|\Psi\left(\cdot, \tau_{j}\right)\right\|^{2} .
\end{aligned}
$$

On the other hand, a slight modification of the argument used in [HV1, Lemma 4.4] yields

$$
I_{2} \leq C\left\|\Psi\left(\cdot, \tau_{j}\right)\right\|^{5 / 2}\left(1+e^{\theta R^{2}}\right)^{5 / 2} e^{5 L / 2} \frac{L}{\left(1-e^{-\varepsilon}\right)^{5 N / 8}}
$$

for some suitable constants $C, \theta$ independent of $\varepsilon, R$, and $\left\{\tau_{j}\right\}$, and (2.4) follows. Taking now the limits $j \rightarrow \infty, R \rightarrow \infty$, and $\varepsilon \rightarrow 0$ (in this order) we obtain that

$$
\lim _{j \rightarrow \infty} \frac{\left\|\omega_{j}(\cdot, \tau)\right\|}{\left\|\Psi\left(\cdot, \tau_{j}\right)\right\|}=0 \quad \text { uniformly for } \tau \in\left[\tau_{j}, \tau_{j}+L\right]
$$

(cf. Lemma 4.5 in [HV1]). The rest of the proof of Lemma 2.3 proceeds then exactly as in [HV $1, \S 4]$. 
As a next step, we shall make use of a result which has been proved in [HV1] for the case $N=1$, and in [FK] for $N>1$. For completeness, we shall give here a different proof along the lines of that in [HV1].

Lemma 2.4. Under our current assumptions, the following alternative holds. Either

$$
\lim _{\tau \rightarrow \infty}\left(\sum_{|\alpha| \neq 2}\left(a_{\alpha}(\tau)\right)^{2}\right)\left(\sum_{|\alpha|=2}\left(a_{\alpha}(\tau)\right)^{2}\right)^{-1}=0
$$

or

$$
|\Psi(\cdot, \tau)|=O\left(e^{-\varepsilon \tau}\right) \text { as } \tau \rightarrow \infty \text { for some } \varepsilon>0 .
$$

Moreover, if (2.5) is satisfied, we have that, if $|\alpha|=2$,

$$
\dot{a}_{\alpha}=\nu_{p} \sum\left\langle H_{\beta} H_{\gamma}, H_{\alpha}\right\rangle a_{\beta} a_{\gamma}+O\left(\varepsilon(\tau) \sum_{|\beta|=2}\left(a_{\beta}(\tau)\right)^{2}\right)
$$

where $\nu_{p}=p(p-1)^{1 /(p-1)} / 2$, summation in the first series is extended to those indexes $\beta, \gamma$ with $|\beta|=|\gamma|=2$, and $\varepsilon(\tau) \rightarrow 0$ as $\tau \rightarrow \infty$.

Proof. Let us define $\rho(\tau)=\|\Psi(\cdot, \tau)\|$. As in [HV1, 55$]$ we have the following possibilities:

(2.8a) $\lim _{\sup } \rightarrow \infty(\tau \rho(\tau))=+\infty$.

(2.8b) There exist $\delta_{0}>0, \delta_{1}>0$ such that

$$
0<\delta_{0} \leq \liminf _{\tau \rightarrow \infty}(\tau \rho(\tau)) \leq \limsup _{\tau \rightarrow \infty}(\tau \rho(\tau)) \leq \delta_{1}<+\infty
$$

(2.8c) $\liminf \operatorname{in}_{\tau \rightarrow \infty}(\tau \rho(\tau))=0$ and $\limsup _{\tau \rightarrow \infty}\left(e^{\varepsilon \tau} \rho(\tau)\right)=+\infty$ for any $\varepsilon>0$.

(2.8d) $\rho(\tau) \leq K e^{-\varepsilon \tau}$ for some $\varepsilon>0, K>0$ and large enough $\tau$.

Clearly, if $(2.8 \mathrm{~d})$ holds, $(2.6)$ is satisfied. On the other hand, if one of the cases $(2.8 \mathrm{a}),(2.8 \mathrm{~b})$, or $(2.8 \mathrm{c})$ takes place, we may argue exactly as in [HV1, $\S 5]$ to obtain that $(2.6)$ holds.

It then remains to show that the Fourier-Hermite coefficients $a_{\alpha}$ with $|\alpha|=2$ satisfy (2.7). We then write

$$
\Psi(y, \tau)=\sum_{|\alpha|=2} a_{\alpha}(\tau) H_{\alpha}(y)+\theta(y, \tau) .
$$

Since $\Psi$ satisfies (1.8), where

$$
f(s)=\frac{p(p-1)^{1 /(p-1)}}{2} s^{2}+g(s) \equiv \nu_{p} s^{2}+g(s),
$$

and $g(s)=O\left(|s|^{3}\right)$ as $s \rightarrow 0$, we readily see that, if $|\alpha|=2$,

$$
\begin{aligned}
a_{\alpha}= & \left\langle f(\Psi), H_{\alpha}\right\rangle \\
= & \nu_{p}\left\langle\left(\sum_{|\beta|=2} a_{\beta}(\tau) H_{\beta}(y)+\theta(y, \tau)\right)^{2}, H_{\alpha}\right\rangle+\left\langle g(\Psi), H_{\alpha}\right\rangle \\
= & \nu_{p}\left\langle\sum_{|\beta|=2} \sum_{|\gamma|=2} a_{\beta}(\tau) a_{\gamma}(\tau) H_{\beta} H_{\gamma}, H_{\alpha}\right\rangle+2 \nu_{p} \sum_{|\beta|=2} a_{\beta}(\tau)\left\langle H_{\beta} \theta, H_{\alpha}\right\rangle \\
& +\nu_{p}\left\langle\theta^{2}, H_{\alpha}\right\rangle+\left\langle g(\Psi), H_{\alpha}\right\rangle .
\end{aligned}
$$


Using the delayed estimates recalled in Lemma 2.1, and arguing as in [HV1, $\S 5]$, we readily bound the last three terms on the right in (2.9) as follows:

$$
\begin{aligned}
\left|\sum_{|\beta|=2} a_{\beta}(\tau)\left\langle H_{\beta} \theta, H_{\alpha}\right\rangle\right| & \leq \sum_{|\beta|=2}\left|a_{\beta}(\tau)\right|\|\theta(\cdot, \tau)\|_{4, w}\left\|H_{\beta}^{2}\right\|_{4 / 3, w}, \\
\left|\left\langle\theta^{2}, H_{\alpha}\right\rangle\right| \leq\|\theta(\cdot, \tau)\|_{4, w}^{2}, & \leq\left|\Psi(\Psi), H_{\alpha}\right\rangle \mid \leq C\|\Psi(\cdot, \tau)\|_{6, w}^{3} \leq C\left\|\Psi\left(\cdot, \tau-\tau^{*}\right)\right\|^{3} \\
& \leq C\|\Psi(\cdot, \tau)\|^{3} \leq C\left(\sum_{|\alpha|=2}\left(a_{\alpha}(\tau)\right)^{2}\right)^{3 / 2}
\end{aligned}
$$

where here and henceforth $C$ will denote a generic constant, possibly changing from line to line. We now claim that

$$
\lim _{\tau \rightarrow \infty} \frac{\|\theta(\cdot, \tau)\|_{4, w}^{2}}{\sum_{|\alpha|=2}\left(a_{\alpha}(\tau)\right)^{2}}=0 .
$$

Indeed, $\theta$ satisfies

$$
\begin{aligned}
\theta_{\tau} & =\Delta \theta-\frac{y \nabla \theta}{2}+\theta+\left[f(\Psi)-\sum_{|\alpha|=2}\left\langle f(\Psi), H_{\alpha}\right\rangle H_{\alpha}\right] \\
& \equiv A_{N} \theta+D(y, \tau)
\end{aligned}
$$

whence, dropping the subscript $N$ for convenience,

$$
\theta(y, \tau)=S_{A}(R) \theta(\cdot, \tau-R)+\int_{\tau-R}^{\tau} S_{A}(\tau-s) D(\cdot, s) d s
$$

for any $R>0$. Recalling (2.5), we see that

$$
\begin{aligned}
\|\theta(\cdot, \tau-R)\| & \ll\left(\sum_{|\alpha|=2}\left(a_{\alpha}(\tau-R)\right)^{2}\right)^{1 / 2} \leq\|\Psi(\cdot, \tau-R)\| \leq C\|\Psi(\cdot, \tau)\| \\
& \leq C\left(\sum_{|\alpha|=2}\left(a_{\alpha}(\tau)\right)^{2}\right)^{1 / 2} \quad \text { as } \tau \rightarrow \infty
\end{aligned}
$$

so that

$$
\begin{aligned}
\left\|S_{A}(R) \theta(\cdot, \tau-R)\right\|_{4, w} & \leq C\|\theta(\cdot, \tau-R)\| \\
& \ll\left(\sum_{|\alpha|=2}\left(a_{\alpha}(\tau)\right)^{2}\right)^{1 / 2} \text { as } \tau \rightarrow \infty .
\end{aligned}
$$

We next set out to estimate the second term on the right in (2.11). To this end, we first notice that, whenever $s \in[\tau-R, \tau]$ and $R>0$ is large enough, 
there holds

$$
\begin{aligned}
\|D(\cdot, s)\|_{5 N, w} & =\left\|f(\Psi)-\sum_{|\alpha|=2}\left\langle f(\Psi), H_{\alpha}\right\rangle H_{\alpha}\right\|_{5 N, w} \\
& \leq\|f(\Psi)\|_{5 N, w}+\sum_{|\alpha|=2}\left|\left\langle f(\Psi), H_{\alpha}\right\rangle\right|\left\|H_{\alpha}\right\|_{5 N, w} \\
& \leq C\|\Psi(\cdot, s)\|_{10 N, w}^{2} \leq C\left\|\Psi\left(\cdot, s-\tau^{*}\right)\right\|^{2} \leq C\|\Psi(\cdot, \tau)\|^{2} \\
& \leq C\left(\sum_{|\alpha|=2}\left(a_{\alpha}(\tau)\right)^{2}\right) .
\end{aligned}
$$

On the other hand, we have that for any $r, q$ with $q>r>1$

(2.14) $\left\|S_{A}(\tau) \phi_{0}\right\|_{r, w} \leq C \frac{e^{C \tau}}{\left(1-e^{-\tau}\right)^{N / 2 q}}\left\|\phi_{0}\right\|_{q, w}$ for some $C>0$ and any $\tau$,

(cf. $[\mathrm{HV} 1, \S 5])$, so that

$$
\begin{aligned}
\int_{\tau-R}^{\tau}\left\|S_{A}(\tau-s) D(\cdot, s)\right\|_{4, w} d s & \leq C e^{C R} \int_{\tau-R}^{\tau} \frac{\|D(\cdot, s)\|_{S N, w}}{\left(1-e^{-(\tau-s)}\right)^{1 / 10}} d s \\
& \leq C\left(\sum_{|\alpha|=2}\left(a_{\alpha}(\tau)\right)^{2}\right) \int_{\tau-R}^{\tau}\left(1-e^{-(\tau-s)}\right)^{-1 / 10} d s \\
& \ll\left(\sum_{|\alpha|=2}\left(a_{\alpha}(\tau)\right)^{2}\right)^{1 / 2}
\end{aligned}
$$

as $\tau \rightarrow \infty$. Putting together (2.11), (2.12), and (2.14), (2.10) follows and the proof is concluded.

We now consider the case where (2.6) is satisfied.

Lemma 2.5. Assume that (2.6) takes place. Then the following alternative holds. Either there exist $m \geq 3$ and constants $c_{\alpha}$ with $|\alpha|=m$, not all identically zero, such that

$$
\left\|\Psi(\cdot, \tau)-\sum_{|\alpha|=m} c_{\alpha} e^{(1-m / 2) \tau} H_{\alpha}\right\|_{H_{w}^{1}}=o\left(e^{(1-m / 2) \tau}\right)
$$

in $H_{w}^{1}\left(\mathbb{R}^{N}\right)$ as $\tau \rightarrow \infty$, or

$$
\Psi(\cdot, \tau) \equiv 0 .
$$

Proof. It can be modeled after that of Proposition 5.8 in [HV1]. Once more, as a starting point we use variation of constants formula in (1.13). This yields

$$
\Psi(y, \tau)=S_{A}(\tau) \Psi\left(\cdot, \tau_{0}\right)+\int_{\tau_{0}}^{\tau} S_{A}(\tau-s) f(\Psi(\cdot, s)) d s
$$

where operator $A_{N}$ is written as $A$ for simplicity. Suppose now that

$$
\|\Psi(\cdot, \tau)\| \leq M e^{-\varepsilon \tau} \text { for some } M>0 \text { and } \varepsilon>0 \text { where }
$$

$$
2 \varepsilon \neq l / 2-1 \text { for } l=3,4, \ldots \text {. }
$$


Fix now a positive integer $k_{0}>2$ such that $k_{0} / 2-1<2 \varepsilon<\left(k_{0}+1\right) / 2-1$. Notice that

$$
\begin{aligned}
\Psi(\cdot, \tau)= & \sum_{|\alpha| \leq k_{0}} a_{\alpha}\left(\tau_{0}\right) e^{(1-|\alpha| / 2)\left(\tau-\tau_{0}\right)} H_{\alpha}(y) \\
& +\sum_{|\alpha| \geq k_{0}+1} a_{\alpha}\left(\tau_{0}\right) e^{(1-|\alpha| / 2)\left(\tau-\tau_{0}\right)} H_{\alpha}(y) \\
& +\sum_{|\alpha| \leq k_{0}} H_{\alpha}(y) \int_{\tau_{0}}^{\tau} e^{(1-|\alpha| / 2)(\tau-s)}\left\langle f(\Psi(\cdot, s)), H_{\alpha}\right\rangle d s \\
& +\sum_{|\alpha| \geq k_{0}+1} H_{\alpha}(y) \int_{\tau_{0}}^{\tau} e^{(1-|\alpha| / 2)(\tau-s)}\left\langle f(\Psi(\cdot, s)), H_{\alpha}\right\rangle d s \\
\equiv & T_{1}+T_{2}+T_{3}+T_{4} .
\end{aligned}
$$

By direct computation, we obtain that

$$
\begin{aligned}
\left\|T_{2}\right\|_{H_{w}^{1}} & \leq\left(\sum_{|\alpha| \geq k_{0}+1}\left(a_{\alpha}\left(\tau_{0}\right)\right)^{2}\right)^{1 / 2}\left(\sum_{|\alpha| \geq k_{0}+1}\left(1+\frac{|\alpha|}{2}\right) e^{2(1-|\alpha| / 2)\left(\tau-\tau_{0}\right)}\right)^{1 / 2} \\
& \leq C\left\|\Psi\left(\cdot, \tau_{0}\right)\right\| \exp \left(\left(1-\frac{k_{0}+1}{2}\right)\left(\tau-\tau_{0}\right)\right) \\
& \leq C\left\|\Psi\left(\cdot, \tau_{0}\right)\right\| \exp \left(-2 \varepsilon\left(\tau-\tau_{0}\right)\right)
\end{aligned}
$$

if $\tau \geq \tau_{0}+1$.

Let us look now at $T_{4}$ in (2.16). Clearly

$$
\begin{aligned}
& \left\|T_{4}(\cdot, \tau)\right\|^{2} \leq \sum_{|\alpha| \geq k_{0}+1}\left(\int_{\tau_{0}}^{\tau} e^{\left(1-\left(k_{0}+1\right) / 2\right)(\tau-s)}\left|\left\langle f(\Psi(\cdot, s)), H_{\alpha}\right\rangle\right| d s\right)^{2} \\
& =\sum_{|\alpha| \geq k_{0}+1}\left(\int_{\tau_{0}}^{\tau} e^{\left(1-\left(k_{0}+1\right) / 2\right) A(\tau-s)} e^{\left(1-\left(k_{0}+1\right) / 2\right) B(\tau-s)}\left|\left\langle f(\Psi(\cdot, s)), H_{\alpha}\right\rangle\right| d s\right)^{2}
\end{aligned}
$$

where $A$ and $B$ are positive numbers such that $A+B=1$. Use of CauchySchwartz inequality yields then

$$
\begin{aligned}
\left\|T_{4}(\cdot, \tau)\right\|^{2}= & \sum_{|\alpha| \geq k_{0}+1}\left(\int_{\tau_{0}}^{\tau} e^{2\left(1-\left(k_{0}+1\right) / 2\right) A(\tau-s)} d s\right) \\
& \times\left(\int_{\tau_{0}}^{\tau} e^{\left(1-\left(k_{0}+1\right) / 2\right) B(\tau-s)}\left|\left\langle f(\Psi(\cdot, s)), H_{\alpha}\right\rangle\right|^{2} d s\right) \\
\leq & C_{1} \int_{\tau_{0}}^{\tau} e^{\left(1-\left(k_{0}+1\right) / 2\right) B(\tau-s)}\|f(\Psi(\cdot, s))\|^{2} d s
\end{aligned}
$$

for some $C_{1}=C_{1}\left(A, k_{0}\right)$. As in [HV1], delayed estimates give $\|f(\Psi(\cdot, s))\|^{2} \leq$ $C e^{-2 \varepsilon s}$, whence

$$
\left\|T_{4}(\cdot, s)\right\|^{2} \leq C_{1} \int_{\tau_{0}}^{\tau} \exp \left(\left(2\left(1-\frac{k_{0}+1}{2}\right) B\right)(\tau-s)-4 \varepsilon s\right) d s .
$$

Therefore, if $B$ is close enough to $1,-4 \varepsilon-2\left(1-\left(k_{0}+1\right) / 2\right) B<0$, and we arrive at

$$
\left\|T_{4}(\cdot, \tau)\right\| \leq C e^{-2 \varepsilon \tau}
$$


whence

$$
\left\|T_{4}(\cdot, \tau)\right\|_{H_{w}^{1}} \leq C e^{-2 \varepsilon \tau} \text { for some } C>0
$$

by standard semigroup theory (cf. for instance Appendix A in [HV1]). On the other hand, since $k_{0} / 2-1<2 \varepsilon$, we readily check that the function

$$
e^{-(1-|\alpha| / 2) s}\left\langle f(\Psi(\cdot, s)), H_{\alpha}\right\rangle
$$

is integrable in $\left[\tau_{0}, \infty\right)$ for $|\alpha| \leq k_{0}$. Thus we can write

$$
\begin{aligned}
\int_{\tau_{0}}^{\tau} e^{-(1-|\alpha| / 2) s}\left\langle f(\Psi(\cdot, s)), H_{\alpha}\right\rangle d s= & \int_{\tau_{0}}^{\tau} e^{-(1-|\alpha| / 2) s}\left\langle f(\Psi(\cdot, s)), H_{\alpha}\right\rangle d s \\
& -\int_{\tau}^{\infty} e^{-(1-|\alpha| / 2) s}\left\langle f(\Psi(\cdot, s)), H_{\alpha}\right\rangle d s \\
\equiv & \beta_{\alpha}-\int_{\tau}^{\infty} e^{-(1-|\alpha| / 2) s}\left\langle f(\Psi(\cdot, s)), H_{\alpha}\right\rangle d s
\end{aligned}
$$

provided that $|\alpha| \leq k_{0}$. In such cases, arguing as in Proposition 5.8 in [HV1] we obtain that

$$
\left|\int_{\tau}^{\infty} e^{-(1-|\alpha| / 2) s}\left\langle f(\Psi(\cdot, s)), H_{\alpha}\right\rangle d s\right| \leq C e^{-2 \varepsilon \tau}
$$

Summing up our previous results, we may rewrite $(2.16)$ in the form

$$
\begin{aligned}
\Psi(y, \tau)= & \sum_{|\alpha| \leq k_{0}}\left(a_{\alpha}+\beta_{\alpha}\right) e^{(1-|\alpha| / 2) \tau} H_{\alpha}(y)+T_{2}+T_{4} \\
& -\sum_{|\alpha| \leq k_{0}} H_{\alpha}(y) \int_{\tau}^{\infty} e^{-(1-|\alpha| / 2) s}\left\langle f(\Psi(\cdot, s)), H_{\alpha}\right\rangle d s .
\end{aligned}
$$

Having obtained (2.18), we then repeat the iteration argument at the end of Proposition 5.8 in [HV1] to conclude. Suppose first that $k_{0} \geq 3$. We then arrive at

$$
\Psi(y, \tau)=\sum_{|\alpha| \leq k_{0}}\left(a_{\alpha}+\beta_{\alpha}\right) e^{(1-|\alpha| / 2) \tau} H_{\alpha}(y)+R(y, \tau)
$$

where $\|R(\cdot, \tau)\|_{H_{w}^{1}}=O\left(e^{-2 \varepsilon \tau}\right)$ for $\tau>1$. Recalling (2.16), we necessarily have $a_{\alpha}+\beta_{\alpha}=0$ for $|\alpha| \leq k_{0}$. Then two possibilities arise. There may be an integer $m \in\left[3, k_{0}\right]$ such that $a_{\alpha}+\beta_{\alpha} \neq 0$ for $|\alpha|=m$ and $a_{\alpha}+\beta_{\alpha}=0$ for $|\alpha|<m$. In this case, we would have

$$
\Psi(\cdot, \tau)=\sum_{|\alpha|=m}\left(a_{\alpha}+\beta_{\alpha}\right) e^{(1-m / 2) \tau} H_{\alpha \alpha}(y)+Q(y, \tau)
$$

where $\|Q(\cdot, \tau)\|_{H_{w}^{\prime}}=o\left(e^{(1-m / 2) \tau}\right)$ as $\tau \rightarrow \infty$ and $(2.15 \mathrm{a})$ holds. If otherwise $a_{\alpha}+\beta_{\alpha}=0$ for any $|\alpha| \in\left[3, k_{0}\right]$, we would obtain

$$
\|\Psi(\cdot, \tau)\|=O\left(e^{-2 \varepsilon \tau}\right) \quad \text { as } \tau \rightarrow \infty
$$

which implies a faster decay than (2.16). Repetition of this argument would lead us to (2.15a) in a finite number of steps, unless the hypotheses in Lemma 2.2 hold, in which case $\Psi \equiv 0$. Finally, if $k_{0}=2$, we would use (2.19) to reduce ourselves to the previous case.

As a next step, we improve the convergence obtained in (2.15a). 
Lemma 2.6. Assume that (2.15a) holds. Then

(a) For any $q \in[1, \infty)$, we have that

$$
\left\|\Psi(\cdot, \tau)-e^{(1-m / 2) \tau} \sum_{|\alpha|=m} c_{\alpha} H_{\alpha}\right\|_{q, w}=o\left(e^{(1-m / 2) \tau}\right) \quad \text { as } \tau \rightarrow \infty .
$$

(b) For any $k \geq 0$ and $\alpha \in(0,1)$,

$$
\Psi(\cdot, \tau)=e^{(1-m / 2) \tau} \sum_{|\alpha|=m} c_{\alpha} H_{\alpha}+o\left(e^{(1-m / 2) \tau}\right)
$$

in $C_{\mathrm{loc}}^{k, \alpha}\left(\mathbb{R}^{N}\right)$ as $\tau \rightarrow \infty$.

Proof. Let us write

$$
\Psi(\cdot, \tau)=\sum_{|\alpha|=m} a_{\alpha}(\tau) H_{\alpha}(\cdot)+\omega(\cdot, \tau) .
$$

Then $\omega(y, \tau)$ solves

$$
\omega_{\tau}=A_{N} \omega+\left[f(\Psi)-\sum_{|\alpha|=m}\left\langle f(\Psi), H_{\alpha}\right\rangle H_{\alpha}\right] \equiv A_{N} \omega+\sigma .
$$

Using variation of constants formula in the equation above, and recalling the argument leading to (2.13) together with (2.14), we obtain

$$
\begin{aligned}
\|\omega(\cdot, \tau)\|_{q, w} & \leq C\left(\|\omega(\cdot, \tau-R)\|+\int_{\tau-R}^{\tau}\left(1-e^{-(\tau-s)}\right)^{-1 / 10}\|\sigma(\cdot, s)\|_{5 N q, w} d s\right) \\
& =o\left(e^{(1-m / 2) \tau}\right) \quad \text { as } \tau \rightarrow \infty
\end{aligned}
$$

provided that $R$ is large enough. On the other hand,

$$
\left\|\sum_{|\alpha|=m} a_{\alpha}(\tau) H_{\alpha}(\cdot)-e^{(1-m / 2) \tau} \sum_{|\alpha|=m} c_{\alpha} H_{\alpha}\right\|_{q, w}=o\left(e^{(1-m / 2) \tau}\right)
$$

as $\tau \rightarrow \infty$, for $1 \leq q<\infty$, whence (2.20) follows.

Hölder continuous convergence requires a different approach. For $R>0$ given, it follows from (2.22) that we may write

$$
\begin{aligned}
\omega(y, \tau)= & \frac{e^{R}}{\left(4 \pi\left(1-e^{-R}\right)\right)^{N / 2}} \int_{\mathbb{R}^{N}} \omega(\lambda, \tau-R) \exp \left(-\frac{\left(y e^{-R / 2}-\lambda\right)^{2}}{4\left(1-e^{-R}\right)}\right) d \lambda \\
& +\int_{\tau-R}^{\tau} \frac{e^{(\tau-s)}}{\left(4 \pi\left(1-e^{-(\tau-s)}\right)\right)^{N / 2}} \int_{\mathbb{R}^{N}} \sigma(\lambda, s) \exp \left(-\frac{\left(y e^{-(\tau-s) / 2}-\lambda\right)^{2}}{4\left(1-e^{-(\tau-s)}\right)}\right) d \lambda .
\end{aligned}
$$

Using Hölder inequality we obtain

$$
\begin{aligned}
|\omega(y, \tau)| \leq & \frac{e^{R}}{\left(4 \pi\left(1-e^{-R}\right)\right)^{N / 2}}\|\omega(\cdot, \tau-R)\|_{q, w} I(y, q, R) \\
& +\int_{\tau-s}^{\tau} \frac{e^{(\tau-s)}}{\left(4 \pi\left(1-e^{-(\tau-s)}\right)\right)^{N / 2}}\|\sigma(\lambda, s)\|_{q, w} I(y, q, \tau-s) d s
\end{aligned}
$$

where

$$
I(y, q, \mu)=\left(\int_{\mathbb{R}^{N}} \exp \left(\frac{q^{\prime} \lambda^{2}}{4 q}-\frac{\left(y e^{-\mu / 2}-\lambda\right)^{2} q^{\prime}}{4\left(1-e^{-\mu}\right)}\right) d \lambda\right)^{1 / q^{\prime}}
$$


and, as usual, $q^{\prime}=q /(q-1)$. Assume now that $q>2$. Then there holds

$$
\frac{\left(\lambda-y e^{-\mu / 2}\right)^{2}}{4\left(1-e^{-\mu}\right)}-\frac{\lambda^{2}}{4 q} \geq \frac{\left(\lambda-y e^{-\mu / 2}\right)^{2}}{8\left(1-e^{-\mu}\right)}+\frac{\left(\lambda-y e^{-\mu / 2}\right)^{2}}{8}-\frac{\lambda^{2}}{4 q} \text {. }
$$

If $|y|<\widetilde{R}$

$$
\frac{\left(\lambda-y e^{-\mu / 2}\right)^{2}}{8}-\frac{\lambda^{2}}{4 q} \geq-C \quad \text { where } C=C(R, q) .
$$

Therefore

$$
|I(y, q, \mu)| \leq\left(e^{C} \int_{\mathbb{R}^{N}} \exp \left(-\frac{\left(\lambda-y e^{-\mu / 2}\right)^{2}}{8\left(1-e^{-\mu}\right)} q^{\prime}\right) d \lambda\right)^{1 / q^{\prime}} \leq C\left(1-e^{-\mu}\right)^{N / 2 q^{\prime}}
$$

and substituting this in $(2.23)$ yields

$$
|\omega(y, \tau)| \leq C\left(\|\omega(\cdot, \tau-R)\|_{q, w}+\int_{\tau-R}^{\tau} e^{(\tau-s) / 2}\left(1-e^{-(\tau-s)}\right)^{\delta}\|\sigma(\cdot, s)\|_{q, w} d s\right)
$$

with $\delta=N / 2 q^{\prime}-N / 2$. We now take $q$ large enough so that $\delta>-1$, and noting that $\|\sigma(\cdot, s)\|_{q, w} \leq C e^{2(1-m / 2) \tau}$, we arrive at $|\omega(y, \tau)|=o\left(e^{(1-m / 2) \tau}\right)$ as $\tau \rightarrow \infty$, uniformly on sets $|y| \leq \widetilde{R}$. Then (2.21) follows by standard regularizing effects for parabolic equations. The case $1<q \leq 2$ follows by Jensen's inequality.

The last result in this section is

Lemma 2.7. Assume that (2.15a) holds. Then $m$ is an even number, and the multilinear form $B(x)=\sum_{|\alpha|=m} c_{\alpha} x^{\alpha}$ is nonnegative definite.

Proof. It is closely related to that of Lemma 2.1 in [HV2]. Set $T=1$ for simplicity. For $0<s<1$, we then define

$$
v_{s}(x, t)=(1-s)^{1 /(p-1)} u\left(x(1-s)^{1 / 2}, s+t(1-s)\right) .
$$

Then $v_{s}$ satisfies

$$
\begin{aligned}
& \left(v_{s}\right)_{t}=\Delta\left(v_{s}\right)+v_{s}^{p} \quad \text { when } x \in \mathbb{R}^{N}, t \in(0,1), \\
& v_{s}(x, 0)=\phi(x,-\log (1-s)) \quad \text { when } x \in \mathbb{R}^{N},
\end{aligned}
$$

where $\phi$ is defined in (1.5). By (2.15a), we have that

$$
\begin{aligned}
v_{s}(x, 0)= & (p-1)^{-1 /(p-1)}-(1-s)^{m / 2-1} \sum_{|\alpha|=m} c_{\alpha} H_{\alpha}(y) \\
& +o\left((1-s)^{m / 2-1}\right) \quad \text { in } H_{w}^{1} \text { as } s \rightarrow 1^{-},
\end{aligned}
$$

where not all the constants $c_{\alpha}$ are zero. Consider now the function

$$
z_{s}(x, t)=\left((S(t) \phi(x,-\log (1-s)))^{-(p-1)}-(p-1) t\right)^{-1 /(p-1)} .
$$

Clearly, $z_{s}(x, 0)=v_{s}(x, 0)$ and it is readily seen that $z_{s}$ is a subsolution of (2.25a), so that

$$
v_{s}(x, t) \geq z_{s}(x, t) .
$$

On the other hand, since

$$
\phi(y, \tau)=(p-1)^{-1 /(p-1)}+\sum_{\tau} a_{r \gamma}(\tau) H_{q}(y)
$$


setting $\tau_{s}=-\log (1-s)$, there holds

$$
S(t) \phi\left(x, \tau_{s}\right)=(p-1)^{-1 /(p-1)}+\sum_{\alpha} a_{\alpha}(\tau)(1-t)^{|\alpha| / 2} H_{\alpha}\left(\frac{x}{(1-s)^{1 / 2}}\right) .
$$

Taking $x=\xi(1-t)^{1 / m}(1-s)^{1 / m-1 / 2}$, we obtain

$$
\begin{aligned}
S(t) \phi( & \left.(1-t)^{1 / m}(1-s)^{1 / m-1 / 2},-\log (1-s)\right) \\
= & (p-1)^{-1 /(p-1)}+\sum_{|\alpha| \neq m} a_{\alpha}\left(\tau_{s}\right)(1-t)^{|\alpha| / m}(1-s)^{|\alpha|(1 / m-1 / 2)} \\
& \times \frac{H_{\alpha}\left(\xi((1-t)(1-s))^{-1 / m-1 / 2}\right)}{((1-t)(1-s))^{|\alpha|(1 / m-1 / 2)}} \\
& +\sum_{|\alpha|=m} a_{\alpha}\left(\tau_{s}\right)(1-t)(1-s)^{1-m / 2} \frac{H_{\alpha}\left(\xi((1-t)(1-s))^{1 / m-1 / 2}\right)}{((1-t)(1-s))^{1-m / 2}}
\end{aligned}
$$

We now relate $t$ and $s$ as follows:

$$
(1-t)=(1-s)^{m / 2-1}
$$

so that $(2.28)$ reads

$$
\begin{aligned}
& S(t) \phi(x,-\log (1-s)) \\
&=(p-1)^{-1 /(p-1)}+\sum_{|\alpha| \neq m} a_{\alpha}\left(\tau_{s}\right)(1-t)^{|\alpha| / 2} H_{\alpha}\left(\frac{x}{(1-t)^{1 / 2}}\right) \\
& \quad+\sum_{|\alpha|=m} a_{\alpha}\left(\tau_{s}\right)(1-t)^{m / 2} H_{\alpha}\left(\frac{x}{(1-t)^{1 / 2}}\right) \\
& \equiv(p-1)^{-1 /(p-1)}+S_{1}+S_{2} .
\end{aligned}
$$

Arguing as in [HV1, Lemma 6.1], we deduce that

$$
S_{1}(x, t)=o(1-t) \text { as } t \rightarrow 1^{-} \text {, uniformly for }|x| \text { bounded. }
$$

Since $a_{\alpha}\left(\tau_{s}\right)=c_{\alpha}(1-t)+o(1-t)$ as $t \rightarrow 1^{-}$, we easily obtain that

$$
S_{2}(x, t)=\left(\sum_{|\alpha|=m} c_{\alpha} x^{\alpha}\right)(1-t)+o(1-t) \text { as } t \rightarrow 1^{-}
$$

uniformly for $|x|$ bounded.

Recalling (2.27), we then have that

$$
\begin{aligned}
& v_{s}(x, t) \\
& \geq\left(\left((p-1)^{-1 /(p-1)}+(1-t) \sum_{|\alpha|=m} c_{\alpha} x^{\alpha}+o(1-t)\right)^{-(p-1)}-(p-1) t\right)^{-1 /(p-1)} \\
& =(1-t)^{-1 /(p-1)}\left((p-1)-(p-1)^{p /(p-1)} \sum_{|\alpha|=m} c_{\alpha} x^{\alpha}+o(1)\right)^{-1 /(p-1)}
\end{aligned}
$$

as $t \rightarrow 1^{-}$, uniformly for $|x|$ bounded. Setting $\tilde{t}=1-(1-s)(1-t)$, we eventually obtain

$$
u\left(\xi(1-t)^{1 / m}, t\right) \geq(1-t)^{-1 /(p-1)}\left((p-1)-(p-1)^{p /(p-1)} B(\xi)+o(1)\right)^{-1 /(p-1)}
$$


as $t \rightarrow 1^{-}$, uniformly for $|\xi|$ bounded. Then if $B(x)=\sum_{|\alpha|=m} c_{\alpha} x^{\alpha}$ is not nonnegative definite (in particular, if $m$ is not even), there would exist $x_{0} \in \mathbb{R}^{N}$ such that

$$
\lim _{t \rightarrow 1^{-}}(1-t)^{1 /(p-1)} u\left(x_{0}(1-t)^{1 / m}, t\right)=+\infty
$$

which contradicts $(1.4 \mathrm{a})$. This concludes the proof.

\section{ANAlysis of the NEUTRAL MODES}

This section is devoted to the study of solutions satisfying (2.5). More precisely, we shall perform a detailed analysis of the ODE system (2.7) which eventually will yield (1.15a). To this end, we begin by introducing some notation. Set

$$
V \equiv \text { linear space spanned by }\left\{H_{\alpha}\right\} \quad \text { with }|\alpha|=2 \text {. }
$$

We then denote by $P$ the orthogonal projection from $L_{w}^{2}\left(\mathbb{R}^{N}\right)$ on $V$. Notice that (2.7) can then be recast as follows:

$$
\begin{array}{r}
\dot{\chi}=\nu_{p} P\left(\chi^{2}\right)+O\left(\varepsilon(\tau)\|\chi\|^{2}\right), \quad \text { where } \chi=P \Psi, \quad \nu_{p}=\frac{p(p-1)^{1 /(p-1)}}{2}, \\
\text { and } \lim _{\tau \rightarrow \infty} \varepsilon(\tau)=0 .
\end{array}
$$

A key point in out approach consists in replacing (3.2) by an evolution equation in a suitable matrix space. Let $M_{N}\left(\mathbb{R}^{N}\right)$ be the linear space of $N \times N$ matrices with real coefficients. For any given $\chi \in V$, we consider $G \in M_{N}\left(\mathbb{R}^{N}\right)$ defined as follows:

$$
G=\left(G_{i, j}\right)
$$

where

$$
G_{i, j}=\left\{\begin{array}{ll}
\sqrt{2}\left\langle\chi, H_{2}\left(y_{i}\right)\right\rangle H_{0}^{N-1} & \text { if } i=j, \\
\left\langle\chi, H_{1}\left(y_{i}\right) H_{1}\left(y_{j}\right)\right\rangle H_{0}^{N-2} & \text { if } i \neq j,
\end{array} \quad 1 \leq i, j \leq N .\right.
$$

Notice that $G$ is symmetric. On the other hand, as recalled in (1.10), we have that $H_{2}\left(y_{i}\right)=c_{2}\left(y_{i}^{2}-2\right), H_{1}\left(y_{i}\right)=c_{1} y_{i}$, and $H_{0}=(4 \pi)^{-1 / 4}$, where $c_{1}=$ $(4 \pi)^{-1 / 4} / \sqrt{2}, c_{2}=c_{1} / 2$. Therefore, $\sqrt{2} c_{2} H_{0}^{N-1}=c_{1}^{2} H_{0}^{N-2}$. A straightforward computation yields then that

$$
G_{i, j}=\beta_{N}\left\langle\chi, y_{i} y_{j}\right\rangle \text { for } i \leq i, j \leq N \text { where } \beta_{N}=c_{1}^{2} H_{0}^{N-2} \text {. }
$$

We also point out that $\chi$ can be reconstructed from the coefficients $G_{i, j}$ by the formula

$$
\chi=\sum_{i=1}^{N} \frac{H_{0}^{N-1}}{\sqrt{2}} G_{i, i} H_{2}\left(y_{i}\right)+\sum_{i<j} H_{0}^{N-2} G_{i, j} H_{1}\left(y_{i}\right) H_{1}\left(y_{j}\right) .
$$

By means of simple (but tedious) computations, equation (3.2) translates into the following set of evolution equations for the coefficients $G_{i, j}$ :

$$
\dot{G}_{i, i}=\sqrt{2} \gamma_{N} \nu_{p} \sum_{l=1}^{N} G_{i, l}^{2}+O\left(\varepsilon(\tau)\|G\|^{2}\right)
$$




$$
\dot{G}_{i, j}=\sqrt{2} \gamma_{N} \nu_{p} \sum_{l=1}^{N} G_{i, l} G_{l, j}+O\left(\varepsilon(\tau)\|G\|^{2}\right)
$$

where for any $B=\left(B_{i, j}\right) \in M_{N}\left(\mathbb{R}^{N}\right),\|B\|^{2}=\sum_{i, j} B_{i, j}^{2}=\operatorname{Tr}\left(B^{2}\right), \nu_{p}$ is given in (3.2), and

$$
\gamma_{N}=\frac{1}{2} A_{2,2,2} A_{0,0,0}^{N-1}=A_{2,1,1} A_{1,1,0} A_{0,0,0}^{N-2}
$$

where for any nonnegative integers $n, m$, and $l$,

$$
A_{n, m, l}=\int_{-\infty}^{+\infty} H_{n}(y) H_{m}(y) H_{l}(y) e^{-y^{2} / 4} d y .
$$

We remark that coefficients $A_{n, m, l}$ have been studied in detail in [HV1]. In particular, $A_{n, m, l} \neq 0$ if and only if $n+m+l$ is even and $n \leq m+l, m \leq n+l$, $l \leq m+n$. In such cases

$$
\begin{aligned}
A_{n, m, l}= & (4 \pi)^{-1 / 4}((n !)(m !)(l !))^{1 / 2} \\
& \cdot\left(\left(\frac{m+n-l}{2}\right) !\left(\frac{n+l-m}{2}\right) !\left(\frac{m+l-n}{2}\right) !\right)^{-1} .
\end{aligned}
$$

We may rewrite (3.5) in the form

$$
\dot{G}=Q(G)+O\left(\varepsilon(\tau)\|G\|^{2}\right) \quad \text { where } Q(G) \equiv \sqrt{2} \gamma_{N} \nu_{p} G^{2} .
$$

An important point to be noticed is the following. The space $V$ defined in (3.1) can be characterized as the space of quadratic polynomials in $\mathbb{R}^{N}$ which are orthogonal in $L_{w}^{2}\left(\mathbb{R}^{N}\right)$ to the affine functions. Therefore $V$ does not depend on any particular choice of axes in $\mathbb{R}^{N}$, and remains invariant under orthogonal transformations. Let $A \in M_{N}\left(\mathbb{R}^{N}\right)$ be any such transformation which maps a coordinate basis $\left(y_{1}, \ldots, y_{N}\right)$ into another one $\left(\tilde{y}_{1}, \ldots, \tilde{y}_{n}\right)$, and let $G$, $\widetilde{G}$ be the corresponding matrices given by (3.3). Then $\widetilde{G}=A G A^{\mathrm{T}}$ where, as usual, $A^{\mathrm{T}}=\left(a_{j, i}\right)$ provided that $A=\left(a_{i, j}\right), 1 \leq i, j \leq N$. It then follows that equations (3.2) are invariant under rotations, and therefore the form of equations (3.5) does not depend on a particular choice of coordinate system. Hence, for any matrix $U \in O(N)$ (the group of orthogonal transformations in $\mathbb{R}^{N}$ ) there holds

$$
U Q\left(U^{\mathrm{T}} \tilde{G} U\right) U^{\mathrm{T}}=Q(\widetilde{G}), \quad \text { where } Q \text { is the quadratic part in (3.6). }
$$

This fact will be repeatedly used in what follows. Notice that (3.7) can be obtained directly by a straightforward calculation.

Since the coefficients $G_{i, j}$ are $C^{1}$, by standard results (cf. for instance [K]) we may define a set of $C^{1}$-functions (not necessarily different) $\lambda_{k}(\tau)$ $(1 \leq k \leq N)$, such that for any fixed $\tau$ the eigenvalues of $G(\tau)$ are given by $\lambda_{1}(\tau), \ldots, \lambda_{N}(\tau)$. We next proceed to obtain the evolution equations for the $\lambda_{k}$ 's.

Lemma 3.1. Assume that (2.5) holds. We then have that for $1 \leq k \leq N$

$$
\dot{\lambda}_{k}=K_{p}\left(\lambda_{k}(\tau)\right)^{2}+O\left(\varepsilon(\tau)\|\lambda(\tau)\|^{2}\right) \text { as } \tau \rightarrow \infty,
$$

where $\lim _{\tau \rightarrow \infty} \varepsilon(\tau)=0,\|\lambda\|^{2}=\sum_{i=1}^{N} \lambda_{i}^{2}$, and $K_{p}=\sqrt{2} \gamma_{N} \nu_{p}$ (cf. (3.5)).

Proof. Fix $\bar{\tau}>0$ large enough. Since $G$ is symmetric, there exists an orthogonal matrix $U(\bar{\tau})$ such that $G(\bar{\tau})=U^{\mathrm{T}}(\bar{\tau}) D(\bar{\tau}) U(\bar{\tau})$, where $D(\bar{\tau})$ is a diagonal 
matrix $D(\bar{\tau}), D(\bar{\tau})=\operatorname{diag}\left\{\lambda_{1}(\bar{\tau}), \ldots, \lambda_{N}(\bar{\tau})\right\}$. We then change to a new coordinate system $\tilde{y}=U(\bar{\tau}) y$. Recalling (3.7), we see that in the new frame the evolution of $\widetilde{G}(\tau)$ is given by

$$
\begin{aligned}
\dot{\tilde{G}}= & U(\bar{\tau}) Q\left(U^{\mathrm{T}}(\bar{\tau}) \widetilde{G}(\tau) U(\bar{\tau})\right) U^{\mathrm{T}}(\bar{\tau}) \\
& +U(\bar{\tau}) O\left(\varepsilon(\tau)\left\|U^{\mathrm{T}}(\bar{\tau}) \widetilde{G}(\tau) U(\bar{\tau})\right\|^{2}\right) U^{\mathrm{T}}(\bar{\tau}) \\
= & Q(\widetilde{G}(\tau))+O\left(\varepsilon(\tau)\|\widetilde{G}(\tau)\|^{2}\right) \equiv Q(\widetilde{G}(\tau))+h(\tau, \bar{\tau})
\end{aligned}
$$

if $\tau>\bar{\tau}$, whereas $\widetilde{G}(\bar{\tau})=D(\bar{\tau})$. Consider now a set of repeated eigenvalues

$$
\lambda_{s_{1}}(\bar{\tau})=\lambda_{s_{2}}(\bar{\tau})=\cdots=\lambda_{s_{r}}(\bar{\tau}) \equiv \bar{\mu}, \quad 1 \leq r \leq N
$$

Let $\left\{e_{1}, \ldots, e_{N}\right\}$ be the canonical basis in $\mathbb{R}^{N}$. By classical perturbation theory for linear operators (see [K, Theorem 5.4, p. 128]), it follows that for $1 \leq j \leq r$, the eigenvalues $\lambda_{s_{j}}(\tau)$ are differentiable at $\tau=\bar{\tau}$ and the $r$-tuple $\left(\dot{\lambda}_{s_{1}}(\bar{\tau}), \ldots, \dot{\lambda}_{s_{r}}(\bar{\tau})\right)$ is given by the eigenvalues of the matrix

$$
H=M(Q(D(\bar{\tau}))+h(\bar{\tau}, \bar{\tau})) M,
$$

where $M$ is the eigenprojection on the space generated by $\left\{e_{i_{1}}, \ldots, e_{i_{r}}\right\}$. Using the precise form of $Q(G)$ (cf. (3.5) and (3.6)), we see that $H=\left(H_{i, j}\right), \quad 1 \leq$ $i, j \leq n$, where

$$
H_{i, j}=\sqrt{2} \gamma_{N} \nu_{p} \bar{\mu}^{2} \delta_{i, j}+\left(e_{s_{i}}, h(\bar{\tau}, \bar{\tau}) e_{s_{j}}\right)
$$

where ( , ) denotes the usual scalar product in $\mathbb{R}^{N}$ and $\delta_{i, j}=1$ if $i=j$ and zero otherwise. By the continuity results in [K, pp. 123-127] it follows that the eigenvalues of $H$ behave as $\sqrt{2} \gamma_{N} \nu_{p} \bar{\mu}^{2}+O\left(\varepsilon(\bar{\tau})\|\lambda(\bar{\tau})\|^{2}\right)$ where $\varepsilon(\bar{\tau})=o(1)$ as $\bar{\tau} \rightarrow \infty$. Applying the same argument for any set of repeated eigenvalues, (3.8) follows. We point out that a careful examination of the previous argument reveals that the continuity result required to obtain the conclusion is the following: For any $\varepsilon>0$, there exists $\delta>0$ such that for any symmetric matrix $A \in M_{r}\left(\mathbb{R}^{r}\right)$ with $\|A\| \leq B<+\infty$, and $\|A-\mu I\| \leq \delta$, the eigenvalues of $A$ satisfy $\left|\lambda_{1}(A)-\mu\right|+\cdots+\left|\lambda_{r}(A)-\mu\right| \leq \varepsilon$. This follows easily from the analysis in $[\mathrm{K}$, loc. cit.].

We next study equations (3.8). As a first step, we obtain

Lemma 3.2. Assume that equation (3.8) holds. Then

$$
\limsup _{\tau \rightarrow \infty} \frac{\lambda_{i}(\tau)}{\left(\sum_{i=1}^{N}\left(\lambda_{i}(\tau)\right)^{2}\right)^{1 / 2}} \leq 0 \text { for } i=1, \ldots, N \text {. }
$$

Proof. Define $\zeta(\tau)=\max \left\{\lambda_{1}(\tau), \lambda_{2}(\tau), \ldots, \lambda_{N}(\tau)\right\}$. Then $\zeta(\tau)$ is absolutely continuous, and by (3.8) we have that

$$
\dot{\zeta}(\tau)=K_{p}(\zeta(\tau))^{2}+\mu(\tau) \quad \text { a.e. } \tau \gg 1
$$

where $|\mu(\tau)| \ll\|\lambda(\tau)\|^{2}, \lambda(\tau) \equiv\left(\lambda_{1}(\tau), \ldots, \lambda_{N}(\tau)\right)$. We shall prove that

$$
\limsup _{\tau \rightarrow \infty} \frac{\zeta(\tau)}{\left(\sum_{i=1}^{N}\left(\lambda_{i}(\tau)\right)^{2}\right)^{1 / 2}} \leq 0 .
$$

Indeed, (3.11) implies (3.10). Assume that (3.11) is not satisfied. Then there exists a sequence $\left\{\tau_{j}\right\}$ such that $\lim _{j \rightarrow \infty} \tau_{j}=\infty$ and

$$
\zeta\left(\tau_{j}\right) /\left\|\lambda\left(\tau_{j}\right)\right\| \geq \delta>0
$$


for some $\delta>0$, which can be assumed to be arbitrarily small. We now claim that

If we select $\delta>0$ sufficiently small in (3.12), then $\zeta(\tau) \geq$ $(\delta / 2)\|\lambda(\tau)\|$ for any $\tau$ large enough.

To show (3.13), we consider the function $S(\tau)=\zeta(\tau)-(\delta / 2)\|\lambda(\tau)\|$. A Liapunov function type argument quite similar, for instance, to that in Lemma 4.8 in [HV1], gives that (3.13) holds as soon as we can prove that

$$
\frac{d S}{d \tau} \geq 0 \quad \text { a.e. in } \frac{\delta}{4}\|\lambda(\tau)\| \leq \zeta(\tau) \leq \delta\|\lambda(\tau)\| .
$$

We now compute

$$
\begin{aligned}
\frac{d S}{d \tau}= & \dot{\zeta}(\tau)-\frac{\delta}{2}\left(\sum_{j=1}^{N} \lambda_{j}(\tau) \dot{\lambda}_{j}(\tau)\right)\left(\sum_{j=1}^{N} \lambda_{j}(\tau)^{2}\right)^{-1 / 2} \\
= & K_{p}(\zeta(\tau))^{2}+\mu(\tau)-\frac{\delta}{2} K_{p}\left(\sum_{j=1}^{N} \lambda_{j}(\tau)^{3}\right)\left(\sum_{j=1}^{N} \lambda_{j}(\tau)^{2}\right)^{-1 / 2} \\
& -\frac{\delta}{2} K_{p}\left(\sum_{j=1}^{N} \lambda_{j}(\tau) \mu_{j}(\tau)\right)\left(\sum_{j=1}^{N} \lambda_{j}(\tau)^{2}\right)^{-1 / 2} \\
\geq & K_{p}(\zeta(\tau))^{2}-o\left(\|\lambda(\tau)\|^{2}\right)-\frac{K_{p} N \delta}{2}(\zeta(\tau))^{3}\|\lambda(\tau)\|^{-1} \text { a.e. as } \tau \rightarrow \infty .
\end{aligned}
$$

Recalling the a priori bounds on $\zeta(\tau)$ assumed in (3.14), we deduce that

$$
\frac{d S}{d \tau} \geq C_{1} \delta^{2}\|\lambda(\tau)\|^{2}-C_{2} \delta^{4}\|\lambda(\tau)\|^{2}+o\left(\|\lambda(\tau)\|^{2}\right) \text { a.e. } \quad \text { as } \tau \rightarrow \infty,
$$

for some positive constants $C_{1}, C_{2}$. Taking $\delta>0$ small enough, (3.14) follows and the proof is concluded.

On the other hand, we clearly have

$$
\liminf _{\tau \rightarrow \infty} \frac{\lambda_{i}(\tau)}{\|\lambda(\tau)\|} \geq-1 \quad \text { for } 1 \leq i \leq N
$$

(3.15b) There exists $j \in\{1, \ldots, N\}$ such that

$$
\liminf _{\tau \rightarrow \infty} \frac{\lambda_{i}(\tau)}{\|\lambda(\tau)\|} \leq-\frac{1}{N}<0
$$

As a further step, we show

Lemma 3.3. Assume that $\liminf _{\tau \rightarrow \infty}\left(\lambda_{i}(\tau) /\|\lambda(\tau)\|\right)=-L<0$ for some $i \in$ $\{1, \ldots, N\}$. Then there exists $\delta>0$ such that

$$
\lambda_{i}(\tau) \leq-\delta\|\lambda(\tau)\| \text { for large enough } \tau \text {. }
$$

Proof. It consists in a Liapunov function type argument, similar to the one recalled in our previous lemma. Let us write

$$
S(\tau)=\lambda_{i}(\tau)^{2}-\sigma\|\lambda(\tau)\|^{2}
$$


where $0<\sigma<L^{2}$ will be selected later. As remarked before, it suffices to show that

$$
d S / d \tau \geq 0
$$

whenever

$$
\sigma / 2 \leq\left(\lambda_{i}(\tau) /\|\lambda(\tau)\|\right)^{2} \leq 3 \sigma / 2 \text { and } \tau \gg 1 .
$$

Recalling (3.8), we readily see that

$$
\frac{d S}{d \tau}=2 \lambda_{i} \dot{\lambda}_{i}-2 \sigma \sum_{j=1}^{N} \lambda_{j} \dot{\lambda}_{j} \geq 2 K_{p}\left(\lambda_{i}(\tau)^{3}-\sigma \sum_{j=1}^{N} \lambda_{j}(\tau)^{3}\right)+o\left(\|\lambda(\tau)\|^{3}\right)
$$

as $\tau \rightarrow \infty$.

Therefore, at any time $\tau=\bar{\tau}$ where (3.17b) holds, we have that

$$
\frac{d S}{d \tau} \geq 2 K_{p}\left(-\frac{27}{8} \sigma^{3}\|\lambda(\bar{\tau})\|^{3}-\sigma \sum_{j=1}^{N} \lambda_{j}(\bar{\tau})^{3}\right)+o\left(\|\lambda(\bar{\tau})\|^{3}\right) \quad \text { as } \bar{\tau} \rightarrow \infty .
$$

Set $J(\tau)=\left\{i \in(1, \ldots, N): \lambda_{i}(\tau)<0\right\}, I(\tau)=\left\{i \in(1, \ldots, N): \lambda_{i}(\tau) \geq 0\right\}$.

We then notice that

$$
\begin{aligned}
& \left(\sum_{j \in I} \lambda_{j}(\tau)^{2}\right)^{3 / 2} \leq N^{1 / 2} \sum_{j \in I} \lambda_{j}(\tau)^{3}, \\
& \sum_{i=1}^{N} \lambda_{j}(\tau)^{3}=-\sum_{j \in J}\left|\lambda_{j}(\tau)\right|^{3}+\sum_{j \in I} \lambda_{j}(\tau)^{3}
\end{aligned}
$$

and, by Lemma 3.2 ,

$$
\lim _{\tau \rightarrow \infty}\left(\sum_{j \in I} \lambda_{j}(\tau)^{3}\right)\|\lambda(\tau)\|^{-1}=0 .
$$

Therefore

$$
\frac{d S}{d \tau}(\bar{\tau}) \geq 2 K_{p} \delta\left(c_{1}-c_{2} \delta^{2}\right)\|\lambda(\bar{\tau})\|^{3}+o\left(\|\lambda(\bar{\tau})\|^{3}\right)
$$

for some positive constants $c_{1}, c_{2}$, and $\bar{\tau}$ large enough. Therefore (3.17) holds and the proof is concluded.

By (3.15b), there exists at least one eigenvalue $\lambda_{k}$ for which (3.16) holds, and therefore (3.8) gives

$$
\dot{\lambda}_{k}=K_{p} \lambda_{\kappa}^{2}+o\left(\lambda_{k}^{2}\right) \quad \text { as } \tau \rightarrow \infty .
$$

Arguing as in [HV1, Proposition 5.7], we obtain for such eigenvalues

$$
\lambda_{k}(\tau)=-1 / K_{p} \tau+o(1 / \tau) \quad \text { as } \tau \rightarrow \infty .
$$

On the other hand, for those $\lambda_{j}$ which do not satisfy (3.15b) we clearly have that $\lim _{\tau \rightarrow \infty} \lambda_{j}(\tau)(\|\lambda(\tau)\|)^{-1}=0$. Taking into account (3.18), we notice that

$$
\|\lambda(\tau)\|=C / \tau+o(1 / \tau) \text { as } \tau \rightarrow \infty \text { for some } C>0 .
$$

Summing up, we have obtained 
Lemma 3.4. There exists $l \in[1, N]$ such that the eigenvalues $\left\{\lambda_{k}(\tau)\right\}$ (after being possibly relabelled) satisfy

$$
\begin{aligned}
& \lambda_{k}(\tau)=-1 / K_{p} \tau+o(1 / \tau) \text { as } \tau \rightarrow \infty \text { for } k=1, \ldots, l, \\
& \lambda_{k}(\tau)=o(1 / \tau) \text { as } \tau \rightarrow \infty \text { for } k=l+1, \ldots, N .
\end{aligned}
$$

To proceed further, we notice that by (3.2) and (3.19) there exists $C>0$ such that $(1 / C)\|G\| \leq\|\chi\| \leq C\|G\|$ and since $\|G\|=\|\lambda\|, C_{1} / \tau \leq\|\chi(\cdot, \tau)\| \leq C_{2} / \tau$ as $\tau \rightarrow \infty$ for some positive constants $C_{1}$ and $C_{2}$. Let us write now

$$
\Psi(y, \tau)=\chi(y, \tau)+\theta(y, \tau), \quad \text { where } \chi \in V, \theta \in V^{\perp}
$$

(cf. (3.11)). We then have

Lemma 3.5. Assume that (2.5) is satisfied, and let $\theta(y, \tau)$ be the function defined in (3.20). Then there holds

$$
\|\theta(\cdot, \tau)\| \leq C / \tau^{2} \text { for some } C>0 \text { as } \tau \rightarrow \infty .
$$

Proof. It consists in a slight modification of the results in [HV4, Lemma 3.1], and [HV1, Proposition 5.8]. The basic idea consists in writing the evolution equation for $\theta(y, \tau)$ and use variation of constants formula to estimate the various terms arising there. To avoid repetition, we shall omit further details, which can be found in the aforementioned papers.

It follows from our previous results that we can recast (3.2) in the form

$$
\dot{\chi}=\nu_{p} P\left(\chi^{2}\right)+O\left(1 / \tau^{3}\right) \quad \text { as } \tau \rightarrow \infty .
$$

Let now $W(\tau)$ be the eigenspace associated to the eigenvalues $\lambda_{1}(\tau), \ldots, \lambda_{l}(\tau)$ satisfying $(3.19 \mathrm{a})$, and let $P_{W(\tau)}$ be the orthogonal projection on $W(\tau)$. Set $R(G, \lambda)=(G-\lambda)^{-1}$. We now prove

Lemma 3.6. There exists $E \equiv \lim _{\tau \rightarrow \infty} P_{W(\tau)}$.

Proof. By classical results (cf. for instance [K, p. 77]) we can write

$$
P_{W(\tau)}=-\frac{1}{2 \pi i} \int_{\Gamma(\tau)} R(G(\tau), \lambda) d \lambda
$$

where $\Gamma(\tau)$ is a closed, positively oriented curve in the complex plane containing $\lambda_{1}(\tau), \ldots, \lambda_{l}(\tau)$ and no other eigenvalues of $G$. By Lemma 3.4, if $\tau$ is large enough, $\Gamma(\tau)$ can be taken to be a ball centered at $\left(-1 / K_{p} \tau\right)$ with radius $1 / 2 K_{p} \tau$. Furthermore, since

$$
\frac{d}{d \tau}(R(G(\tau), \lambda))=-R(G(\tau), \lambda) \dot{G}(\tau) R(G(\tau), \lambda)
$$

(cf. for instance [K, p. 32]), using the analyticity properties of the resolvent $R$ and (3.6), we obtain that

$$
\begin{aligned}
\frac{d}{d \tau}\left(P_{W(\tau)}\right)= & \frac{1}{2 \pi i} \int_{\Gamma(\tau)} R(G(\tau), \lambda) \dot{G}(\tau) R(G(\tau), \lambda) d \lambda \\
= & \frac{1}{2 \pi i} \int_{\Gamma(\tau)} R(G(\tau), \lambda) Q(G(\tau)) R(G(\tau), \lambda) d \lambda \\
& +\frac{1}{2 \pi i} \int_{\Gamma(\tau)} R(G(\tau), \lambda) h(\tau) R(G(\tau), \lambda) d \lambda \\
\equiv & I_{1}+I_{2},
\end{aligned}
$$


where $\|h(\tau)\|=O\left(1 / \tau^{3}\right)$ as $\tau \rightarrow \infty$ by (3.22). We know that, for any fixed $\tau_{0}$, there exists an orthogonal matrix $U\left(\tau_{0}\right)$ such that $G\left(\tau_{0}\right)=U^{\mathrm{T}}\left(\tau_{0}\right) D\left(\tau_{0}\right) U\left(\tau_{0}\right)$ where $D\left(\tau_{0}\right)$ is diagonal. Therefore

$$
\begin{aligned}
& R\left(G\left(\tau_{0}\right), \lambda\right) Q\left(G\left(\tau_{0}\right)\right) R\left(G\left(\tau_{0}\right), \lambda\right) \\
& \quad=U^{\mathrm{T}}\left(\tau_{0}\right) R\left(D\left(\tau_{0}\right), \lambda\right) Q\left(D\left(\tau_{0}\right)\right) R\left(D\left(\tau_{0}\right), \lambda\right) U\left(\tau_{0}\right)
\end{aligned}
$$

and since

$$
R\left(D\left(\tau_{0}\right), \lambda\right) Q\left(D\left(\tau_{0}\right)\right) R\left(D\left(\tau_{0}\right), \lambda\right)=\operatorname{diag}\left(K_{p} \lambda_{i}^{2} /\left(\lambda_{i}-\lambda\right)^{2}\right)
$$

where $K_{p}$ is given in (3.8), we readily see that $I_{1}=0$.

On the other hand, for $\tau_{0} \gg 1$ and $\lambda \in \Gamma\left(\tau_{0}\right)$, Lemma 3.4 yields that $\left\|R\left(G\left(\tau_{0}\right), \lambda\right)\right\| \leq C \tau_{0}$ for some $C>0$. Recalling the bound available for $\|h(\cdot, \tau)\|$, we obtain

$$
\left|\frac{d}{d \tau} P_{W\left(\tau_{0}\right)}\right| \leq\left|I_{2}\right| \leq \frac{C_{1}}{\tau_{0}} \int_{\Gamma\left(\tau_{0}\right)}|d \lambda| \leq \frac{C_{2}}{\tau_{0}^{2}}
$$

for some positive constants $C_{1}$ and $C_{2}$, which implies the result.

It is worth pointing out that the operator $E$ obtained in Lemma 3.6 is an orthogonal projection operator on an $l$-dimensional space, since the same happens for $W(\tau)$ when $\tau$ is large enough (see for instance [K, pp. 33-35, 58-60]). As a final step, we next show

\section{Lemma 3.7. There holds}

$$
\lim _{\tau \rightarrow \infty}(\tau G(\tau))=-E / K_{p}
$$

where $C_{p}$ is given in (3.5).

Proof. Let us write

$$
\begin{aligned}
G(\tau)= & P_{W(\tau)} G(\tau) P_{W(\tau)}+P_{W(\tau)} / K_{p} \tau \\
& +\left(I-P_{W(\tau)}\right) G(\tau)\left(I-P_{W(\tau)}\right) P_{W(\tau)} / K_{p} \tau .
\end{aligned}
$$

Notice that $\left(I-P_{W(\tau)}\right)$ is the orthogonal projection on the eigenspace corresponding to the eigenvalues $\lambda_{l+1}(\tau), \ldots, \lambda_{N}(\tau)$. Then, by Lemma 3.4

$$
\left\|\left(I-P_{W(\tau)}\right) G(\tau)\left(I-P_{W(\tau)}\right)\right\|=o(1 / \tau) \quad \text { as } \tau \rightarrow \infty .
$$

On the other hand,

$$
\begin{aligned}
& \left\|P_{W(\tau)} G(\tau) P_{W(\tau)}+P_{W(\tau)} / K_{p} \tau\right\|^{2} \\
& \quad=\operatorname{Trace}\left\{\left(P_{W(\tau)} G(\tau) P_{W(\tau)}+P_{W(\tau)} / K_{p} \tau\right)^{2}\right\} \\
& \quad=\sum_{k=1}^{l}\left(\lambda_{k}(\tau)+\frac{1}{K_{p} \tau}\right)^{2}=o\left(\frac{1}{\tau^{2}}\right) \text { as } \tau \rightarrow \infty .
\end{aligned}
$$

Then (3.24) yields

$$
G(\tau)=-P_{W(\tau)} / K_{p} \tau+o(1 / \tau) \quad \text { as } \tau \rightarrow \infty .
$$

Recalling Lemma 3.7. the result follows. 
End of the proof of the theorem. Since $E$ is an orthogonal projection on an $l$-dimensional space, $E$ can be written in a suitable system of coordinates in the form

$$
E=\left(\begin{array}{ccccccccc}
1 & & & & & & & & \\
& \cdot & & & & & & & \\
& & \cdot & & & & & & \\
& & \cdot & & & & & & \\
& & & 1 & & & & \\
& & & & 0 & & & \\
& & & & & \cdot & & \\
& & & & & & \cdot & \\
& & & & & & & \cdot & \\
& & & & & & & & 0
\end{array}\right)
$$

where there are $N-l$ zeroes in the main diagonal. In such a coordinate system, Lemma 3.7 yields that

$$
\begin{aligned}
& G_{i, i}=-1 / K_{p} \tau+o(1 / \tau) \quad \text { as } \tau \rightarrow \infty \quad \text { if } 1 \leq i \leq l, \\
& G_{i, j}=o(1 / \tau) \text { as } \tau \rightarrow \infty \quad \text { otherwise. }
\end{aligned}
$$

By the very definition of $G$, this means that

$$
\left\|\Psi(\cdot, \tau)+\frac{C_{p}}{\tau} \sum_{k=1}^{l} H_{2}\left(y_{k}\right)\right\|=o\left(\frac{1}{\tau}\right) \quad \text { as } \tau \rightarrow \infty,
$$

where $C_{p}$ is given in $(1.15 \mathrm{a})$. Convergence in $H_{w}^{1}\left(\mathbb{R}^{N}\right)$ follows then by standard regularizing effects for semigroup evolution equations. Finally, convergence in $C_{\text {loc }}^{k, \alpha}\left(\mathbb{R}^{N}\right)$ can be obtained as in Lemma 2.6.

\section{REFERENCES}

[AW] D. G. Aronson and H. F. Weinberger, Multidimensional nonlinear diffusion arising in population genetics, Adv. Math. 30 (1978), 33-76.

[BBE] J. Bebernes, A. Bressan, and D. Eberly, A description of blow-up for the solid fuel ignition model, Indiana Univ. Math. J. 36 (1987), 131-136.

[BB] J. Bebernes and S. Bricher, Final time blow-up profiles for semilinear parabolic equations via center manifold theory (to appear).

[BE] J. Bebernes and D. Eberly, Mathematical problems from combustion theory, Applied Mathematical Sciences, vol. 83, Springer-Verlag, Berlin, Heidelberg, and New York, 1989.

[B] A. Bressan, On the asymptotic shape of blow-up, Indiana Univ. Math. J. 39 (1990), 947-960.

[CM] X. Y. Chen and H. Matano, Convergence, asymptotic periodicity and finite point blow-up in one-dimensional semilinear heat equations, J. Differential Equations 78 (1989), 160-190.

[FK] S. Filippas and R. V. Kohn, Refined asymptotics for the blow-up of $u_{t}-\Delta u=u^{p}$, Comm. Pure Appl. Math. 45 (1992), 821-869.

[FL] S. Filippas and W. Liu, On the blow-up of multidimensional semilinear heat equations, Ann. Inst. H. Poincaré (to appear).

[FM] A. Friedman and J. B. McLeod, Blow-up of positive solutions of semilinear heat equations, Indiana Univ. Math. J. 34 (1985), 425-447.

[Fu] H. Fujita, On the blowing-up of solutions of the Cauchy problem for $u_{t}=\Delta u+u^{1+\alpha}$, J. Fac. Sci. Univ. Tokyo Sect. IA Math. 13 (1966), 109-124.

[GK1] Y. Giga and R. V. Kohn, Asymptotically self-similar blow-up of semilinear heat equations, Comm. Pure Appl. Math. 38 (1985), 297-319. 
[GK2] _ Characterizing blow-up using similarity variables, Indiana Univ. Math. J. 36 (1987), $1-40$.

[GK3] __ Nondegeneracy of blow-up for semilinear heat equations, Comm. Pure Appl. Math. 42 (1989), 845-884.

[GP] V. A. Galaktionov and S. A. Posashkov, Application of new comparison theorems in the investigation of unbounded solutions of nonlinear parabolic equations, Differentsial'nge Uravneniya 22 (1986), 1165-1173.

[HV1] M. A. Herrero and J. J. L. Velázquez, Blow up behaviour of one dimensional semilinear parabolic equations, Ann. Inst. H. Poincaré (to appear).

[HV2] __ Flat blow-up in one-dimensional semilinear heat equations, Differential Integral Equations 5 (1992), 973-998.

[HV3] _ Blow-up profiles in one-dimensional, semilinear parabolic problems, Comm. Partial Differential Equations 17 (1992), 205-219.

[HV4] __ Generic behaviour of one-dimensional blow-up patterns, Ann. Scuola Norm. Sup. Pisa (to appear).

[HV5] _- Plane structures in thermal runaway, Israel J. Math. (to appear).

[K] T. Kato, A short introduction to perturbation theory for linear operators, Springer-Verlag, Berlin, Heidelberg, and New York, 1982.

[L] A. A. Lacey, The form of blow-up for nonlinear parabolic equations, Proc. Roy. Soc. Edinburgh Sect. A 98 (1984), 183-202.

[Li] W. Liu, Blow-up behaviour for semilinear heat equations: multi-dimensional case, Rocky Mountain J. Math. (to appear).

[W] F. B. Weissler, Single point blow-up for a general semilinear heat equation, Indiana Univ. Math. J. 34 (1983), 881-913.

Departamento de Matemática Aplicada, Facultad de Ciencias Matemáticas, UniverSidAD COMPLUtense, 28040 MAdRID, Spain 